\title{
Razonamiento explicativo y evolución de lógicas: Una aproximación desde la semántica de mundos posibles*
}

\section{Explanatory reasoning and evolution of logics: An approach from possible worlds semantics}

\author{
FERNANDO SOLER TOSCANO \\ Grupo de Lógica, Lenguaje e Información. Universidad de Sevilla (España)
}

Recibido: 14-6-2012 Aprobado definitivamente: 17-10-2012

RESUMEN

Realizamos un acercamiento al razonamiento explicativo mediante estructuras modales. Usamos el formalismo bien conocido de los marcos de Kripke, pero asociamos a cada mundo, no una interpretación, sino una lógica. De este modo, definimos operadores que nos permiten expresar distintas modificaciones que puede sufrir una teoría, concretamente ampliaciones y contracciones. Mostramos cómo los tratamientos lógicos tradicionales del razonamiento abductivo pueden ser comprendidos desde nuestra propuesta.

\section{PALABRAS CLAVE \\ RAZONAMIENTO ABDUCTIVO, LÓGICAS NO CLÁSICAS, LÓGICA MODAL, MODELOS DE KRIPKE, EXPLICACIÓN CIENTÍFICA.}

\section{ABSTRACT}

We propose an approach to explanatory reasoning through modal structures. We use the wellknown formalism of Kripke frames, but associated with each world, there is not an interpretation but a logic. Then, we define operators that allow us to express different modifications of a theory, specially expansions and contractions. We show that traditional logical treatments of abductive reasoning can be understood from our proposal.

\section{KEYWORDS}

\section{ABDUCTIVE REASONING, NON-CLASSICAL LOGICS, MODAL LOGIC, KRIPKE FRAMES, SCIENTIFIC EXPLANATION.}

\footnotetext{
* El presente artículo se ha realizado con el apoyo de los proyectos Interpretaciones Alternativas de Lógicas no Clásicas (Junta de Andalucía, P10-HUM-5844) y Conciencia, Lógica y Computación (Ministerio de Ciencia e Innovación, FFI2011-29609-C02-01)
} 


\section{INTRODUCCIÓN}

La abducción es un tipo de inferencia donde, a partir de una observación novedosa, se obtiene una explicación plausible. Un posible esquema para caracterizar el razonamiento abductivo es el siguiente:

$$
\frac{\phi, \quad \alpha \rightarrow \phi}{\alpha}
$$

Este esquema es incorrecto desde el punto de vista deductivo, ya que la verdad de $\alpha$ no puede inferirse necesariamente de la verdad de $\phi$ y $\alpha \rightarrow \phi$, pues se trata de la conocida falacia de afirmación del consecuente. Sin embargo, en ciertos contextos, $\alpha$ puede ser una buena explicación del hecho sorprendente $\phi$, dado que sabemos que $\alpha \rightarrow \phi$, es decir, que $\alpha$ es condición suficiente de $\phi$. Son numerosas las aplicaciones que el razonamiento abductivo encuentra en disciplinas como la inteligencia artificial, la lingüística o la epistemología. En filosofía de la ciencia, la abducción se ha llegado a ver incluso como la lógica del descubrimiento científico.

El esquema que hemos proporcionado más arriba se usa típicamente para simbolizar el razonamiento abductivo, aunque debemos incluir un elemento fundamental: la teoría base. En efecto, un problema abductivo (la aparición de un hecho sorprendente que requiere una explicación) aparece siempre dentro de una teoría $\Theta$ que formaliza nuestro conocimiento sobre algún dominio específico. ${ }^{1}$ Formalmente, decimos que $\langle\Theta, \phi\rangle$ es un problema abductivo sii (si y solo si):

1. $\Theta \not \models \phi$

2. $\Theta \not \models \neg \phi$

Con estas dos condiciones estamos exigiendo que ni $\phi$ ni su negación sean consecuencia lógica de $\Theta$. Esta definición se maneja, con pequeñas diferencias, en los distintos acercamientos formales al razonamiento abductivo. Ver por ejemplo (M.C. Mayer y F. Pirri 1993) o (A. Aliseda 2006).

\footnotetext{
${ }^{1}$ Entendemos una teoría como un conjunto de fórmulas del lenguaje que se trate. En ningún caso contemplamos que estemos ante teorías completas, a partir de las cuales no cabe plantear problema abductivo alguno. En general, una teoría $\Theta$ es completa si para toda fórmula $\phi$ del lenguaje correspondiente $\phi$ o $\neg \phi$ son consecuencia lógica de $\Theta$ (Zabalardo 2002). Tampoco es necesario considerar teorías cerradas bajo consecuencia lógica, es decir, aquellas tales que para cada fórmula $\alpha$, si $\Theta \vDash \alpha$ entonces $\alpha \in \Theta$.
} 
Aparentemente, en la consideración de un problema abductivo aparecen solo $\Theta$ y $\phi$, la teoría base y el hecho sorprendente, respectivamente. Ahora bien, de forma implícita también es relevante $\vDash$, la relación de consecuencia lógica que se maneja, que en cada caso permitirá establecer qué es y qué no es un problema abductivo. De hecho en (A. Aliseda 2006; pp. 4647) se señala el parámetro inferencial como tercer elemento a tener en cuenta en la abducción y se indica que no hay ninguna razón que obligue a que la lógica en cuestión haya de ser la lógica clásica. No obstante, y dado que es habitual en diversos ámbitos trabajar con la lógica clásica, o con extensiones de la misma, se ha venido asumiendo la relación de consecuencia lógica clásica. Por nuestra parte vamos a subrayar la importancia que tiene la elección de una relación de consecuencia lógica, dado que caracteriza la lógica subyacente en cierto contexto. La relación de consecuencia elegida determinará las prácticas inferenciales que rigen los razonamientos propios del contexto de que se trate, y por esta razón se convierte en un elemento clave en la formalización del razonamiento abductivo. Pero no nos constreñiremos a la relación de consecuencia lógica clásica.

Consideramos que, así como podemos resolver un problema abductivo $\langle\Theta, \phi\rangle$ mediante la extensión de la teoría base $\Theta$ (que es lo que plantean las definiciones anteriores: añadir $\alpha$ a $\Theta$ para que se verifique $\Theta \cup\{\alpha\} \vDash \phi$ ), también podremos modificar la relación de consecuencia lógica utilizada $\vDash$ de forma que con una nueva relación de consecuencia $\vDash^{\prime}$ se verifique $\Theta \vDash^{\prime} \phi$. De este modo resolvemos el problema abductivo $\langle\Theta, \phi\rangle$ extendiendo la lógica subyacente. Este planteamiento nos permitirá abordar otras clases de problemas abductivos, no resolubles mediante extensión de teorías sino, más bien, introduciendo cambios en los postulados lógicos, es decir, cambios de lógica. Aunque cada cambio de lógica requiere abordajes específicos, ${ }^{2}$ nuestro tratamiento metateórico será lo suficientemente flexible, como veremos, para adaptarse a distintas situaciones, ya que se situará en el nivel en que evolucionan las lógicas, y no en el de las diferentes lógicas que evolucionan. Por otra parte, la evolución de teorías es un tema del máximo interés en epistemología. Por mencionar solo algún caso, baste recordar el modelo AGM (C.E. Alchourrón et al. 1985) de cambio epistémico, o los planteamientos propios de la concepción estructural de las teorías

\footnotetext{
${ }^{2}$ Por ejemplo, las ideas presentadas en este trabajo se pueden aplicar al contexto de la lógica modal, de forma que podremos resolver un problema abductivo modificando las propiedades estructurales de la relación de accesibilidad entre mundos posibles.
} 
científicas. En el presente trabajo, a través de una modificación en la semántica de mundos posibles, proporcionamos un nuevo acercamiento al problema de la evolución de teorías que puede compararse con los enfoques tradicionales.

Como hemos indicado, la relación de consecuencia lógica juega un papel fundamental en la caracterización de los problemas abductivos. Pero también de sus soluciones. Así, decimos que $\alpha$ es una solución al problema abductivo $\langle\Theta, \phi\rangle$ sii:

1. $\Theta \cup\{\alpha\} \vDash \phi$

2. $\Theta \cup\{\alpha\} \not \models \perp$

3. $\{\alpha\} \not \models \phi$

La condición (1) establece que el hecho a explicar pase a ser consecuencia lógica de la teoría extendida. Con (2) se exige que la explicación sea consistente con el conocimiento anterior $\Theta$ y, con (3) que la explicación no sea tan fuerte que baste para explicar ella sola $\phi$ sin el concurso de la teoría base $\Theta$, ya que entonces sería una explicación externa a la teoría, mientras que se busca explicar $\phi$ dentro de la teoría $\Theta$. En (A. Aliseda 2006) se llama soluciones abductivas planas a las que cumplen solo la condición (1), consistentes a las que cumplen (1) y (2) y explicativas las que verifican (1) y (3). Nosotros trabajaremos con la abducción consistente explicativa (que requiere las tres condiciones anteriores), a la que Aliseda otorga un mayor valor epistémico, dado que es la única que garantiza la eliminación de trivialidades del conjunto de soluciones abductivas posibles.

Para resolver problemas abductivos se han propuesto diversos formalismos, tratando de capturar lo que habitualmente se conoce como la inferencia de la mejor explicación (P. Lipton 1991). Ahora bien, la abducción lógica se queda corta si solo puede dar cuenta de pequeños añadidos a las teorías (generalmente, además, solo funciona bien en lógica proposicional, insuficientemente expresiva para representar teorías de cierta complejidad). De hecho, los tratamientos lógicos del razonamiento abductivo reciben constantemente críticas desde la epistemología, por no ser capaz de modelar modificaciones profundas en las teorías, como las que conllevan los cambios de paradigma.

La tesis de comprehensión (J. Hintikka 1998) exige que el razonamiento abductivo incluya todas las operaciones por las que se generan nuevas teorías. Por ello, en este trabajo proponemos un acercamiento formal al razonamiento abductivo que permite no solo la 
inferencia de nuevos hechos (abducción lógica tradicional), sino que puede explicar incluso modificaciones en la lógica subyacente a ciertas teorías. Solemos resolver un problema abductivo añadiendo nuevas fórmulas a $\Theta$ pero, ¿por qué no modificar nuestra lógica? Hay ciertos episodios bien conocidos por los historiadores de la ciencia, como el paso de la mecánica clásica a la mecánica cuántica, que sería incorrecto afirmar que se reducen a añadir nuevos postulados a la teoría, o abandonar algunos de los antiguos. Cambia toda una concepción de la realidad, que conlleva una nueva forma de razonar con ella. Más concretamente, los primeros planteamientos de Birkhoff y von Neumann, ya en 1936, tratan de hallar la lógica subyacente de la mecánica cuántica y sugieren que «la transición de la mecánica clásica a la mecánica cuántica involucra el paso de un cálculo proposicional clásico a un cálculo proposicional con estructura no clásica» (S. F. Martínez 1995; p. 228). Otro botón de muestra de cambio de perspectiva lógica, en este caso con elementos de la semántica en sentido general de Henkin, es la fundamentación del análisis no estándar en un modelo de orden superior no estándar de universo $* \mathbb{R}$, un campo ordenado que es no arquimediano, pues contiene números que son mayores que todos los números de $\mathbb{R}$, el universo estándar de los reales. A partir de las características de $* \mathbb{R}$ se llega a establecer con precisión la noción de inifinitésimo (A. Robinson 1966).

\section{MODELOS EXPLICATIVOS}

En la concepción estructural de las teorías científicas se parte de la noción de núcleo teórico, tupla que comprende un conjunto de modelos potenciales, un conjunto de modelos parciales, un conjunto de modelos, y las llamadas «condiciones de ligadura». Cada teoría científica estará integrada por un núcleo teórico y un conjunto de aplicaciones propuestas. Son definibles redes y, mediante el concepto de evolución teórica progresiva, se trata dar cuenta de cómo evolucionan las teorías (J. A. Díez y C. U. Moulines 1997).

Para tratar evoluciones teóricas como las propuestas en la concepción estructural con herramientas lógicas, vamos a emplear nociones provenientes de la semántica kripkeana de mundos posibles para la lógica modal. Pero asignaremos a cada mundo, no una interpretación, sino una lógica, es decir, una teoría, entendida ahora no solo como un conjunto de postulados, sino que igualmente incluimos en ella las reglas que nos permitirán razonar con los datos, es decir, una relación de consecuencia lógica propia para cada mundo. Definimos una relación 
de accesibilidad $\mathfrak{R}$ entre mundos que nos indica cuándo, desde una lógica, podemos pasar a otra (cuándo desde una teoría podemos evolucionar a otra). Así, si desde una teoría $w$ resulta accesible otra teoría $w^{\prime}$, es decir $\mathfrak{R} w w^{\prime}$, entonces la teoría $w$ podría evolucionar hasta $w^{\prime}$, modificándose en su caso no solo el conjunto de proposiciones que conforman la teoría, sino igualmente su lógica subyacente, lo que puede incluir cuestiones profundas como el carácter clásico o no clásico de las inferencias. Téngase en cuenta que las lógicas que caracterizan cada mundo podrán ser completamente diferentes. Esto significa que las condiciones para que cierta fórmula $\beta$ sea consecuencia de un conjunto $\Gamma$ de fórmulas dado pueden diferir completamente entre un mundo y otro. En un mundo podemos usar lógica clásica, en otro lógica no monótona, etc.

Formalmente, un modelo explicativo es una tupla:

$$
M=\langle L, W, \Lambda, \mathfrak{R}, \pi\rangle
$$

donde:

- $\quad L$ es un lenguaje formal, que tomamos como lenguaje base, común a todos los mundos. $^{3}$

- $\quad W$ es un conjunto de mundos no vacío y enumerable.

- $\Lambda$ es un conjunto no vacío de lógicas sobre el lenguaje $L$. $^{4}$

- $\mathfrak{R} \subseteq W \times W$ es la relación de accesibilidad entre mundos.

- $\pi: W \mapsto \Lambda$ es una función que asigna a cada mundo $w \in W$ una lógica $\pi(w) \in \Lambda$.

Establecido $M$, definimos $L_{M}$ a partir del lenguaje base $L$. Los símbolos $\sim, \&, \nabla, \Rightarrow, \diamond^{+}$, $\diamond^{-}, \square^{+}, \square^{-}$, son específicos de $L_{M}$, que se define como el conjunto más pequeño que verifica:

1. Si $\alpha \in L$, entonces $\alpha \in L_{M}$

2. Si $\alpha \in L_{M}$, entonces $\sim \alpha \in L_{M}$

3. Si $\alpha, \beta \in L_{M}$, entonces $\alpha \nabla \beta, \alpha \& \beta, \alpha \Rightarrow \beta \in L_{M}$

\footnotetext{
${ }^{3}$ Por simplicidad en la presentación, tomamos un lenguaje común a todos los mundos. Podríamos, sin embargo, considerar un lenguaje $L_{w}$ para cada mundo $w \in W$ y establecer mecanismos de traducción de fórmulas de un lenguaje a otro.

${ }^{4}$ Cada elemento $w_{i} \in W$ tendrá la forma $\left\langle L, \vDash_{w_{i}}\right\rangle$, donde $\vDash_{w_{i}}$ es una relación de consecuencia lógica (no necesariamente la clásica) sobre $L$, es decir, $\vDash_{w_{i}} \subseteq \wp(L) \times L$.
} 
4. Si $\alpha \in L_{M}$, entonces $\diamond^{+} \alpha, \diamond^{-} \alpha, \square^{+} \alpha, \square^{-} \alpha \in L_{M}$

El lenguaje $L_{M}$ del modelo explicativo funciona como un metalenguaje que se construye sobre el lenguaje base $L$. Podemos dar una semántica para $L_{M}$ que, aunque dependerá de la semántica propia de la lógica de cada mundo, se puede definir independientemente. A cada fórmula $\varphi \in L_{M}$ asignamos un conjunto $\|\varphi\| \subseteq W$ de mundos que satisfacen $\varphi$. Escribimos, indistintamente, $w \in\|\varphi\| \mathrm{y}\langle M, w\rangle \mid=\varphi$ para indicar que el mundo $w$ (que representa una teoría cuya lógica subyacente es $\pi(w) \in \Lambda$ ) perteneciente al modelo explicativo $M$ satisface $\varphi$. El conjunto $\|\varphi\|$ se define inductivamente:

1. $\|\varphi\|=\left\{w \in W \mid \vDash_{w} \varphi\right\}$, si $\varphi \in L$

2. $\|\sim \alpha\|=W-\|\alpha\|$

3. $\|\alpha \& \beta\|=\|\alpha\| \cap\|\beta\|$

4. $\|\alpha \nabla \beta\|=\|\alpha\| \cup\|\beta\|$

5. $\|\alpha \Rightarrow \beta\|=\|\sim \alpha\| \cup\|\beta\|$

6. $\left\|\square^{+} \alpha\right\|=\{w \in W \mid \forall v$, si $\mathfrak{R} w v$ entonces $v \in\|\alpha\|\}$

7. $\left\|\square^{-} \alpha\right\|=\{w \in W \mid \forall v$, si $\mathfrak{R} v w$ entonces $v \in\|\alpha\|\}$

8. $\left\|\triangleright^{+} \alpha\right\|=\{w \in W \mid \exists v, \mathfrak{R} w v$ y $\left.v \in\|\alpha\|)\right\}$

9. $\left\|\diamond^{-} \alpha\right\|=\{w \in W \mid \exists v, \mathfrak{R} v w$ y $v \in\|\alpha\|\}$

La cláusula básica (1) indica para cada fórmula $\varphi$ del lenguaje base $L$ el conjunto de mundos que la satisfacen. Son todos los mundos $w \in W$ tales que $\varphi$ es válida en la lógica $\pi(w)$. La semántica de las conectivas $\sim, \&, \nabla \mathrm{y} \Rightarrow$ se especifica en (2)-(5). Operan, respectivamente, como la negación, conjunción, disyunción e implicación. ${ }^{5}$ Los mundos donde se satisface $\square^{+} \alpha$ son, tal como indica (6), aquellos desde los cuales solo se puede acceder a mundos que

\footnotetext{
${ }^{5}$ No confundir con los operadores proposicionales que puedan existir en $L$. Los operadores presentados se aplican a fórmulas de $L_{M}$ y su semántica es la que estamos definiendo. Los operadores proposicionales que puedan existir en $L$ solo se aplican a fórmulas de $L$, y en la medida en que lo permita la sintaxis de este lenguaje. Además, la semántica de los operadores de $L$ la determinará cada mundo en función de su lógica asociada.
} 
satisfacen $\alpha$. En (7) se define el conjunto de mundos donde se satisface $\square^{-} \alpha$ como aquellos a los que solo se puede acceder desde otros que satisfacen $\alpha$. Finalmente, (8) y (9) definen la semántica de los operadores existenciales $\diamond^{+}$y $\diamond^{-}$. Se puede observar que $\square^{+}$y $\diamond^{+}$son los operadores estándar modales interpretados en $\mathfrak{R}$, así como $\square^{-}$y $\diamond^{-}$son interpretados en $\mathfrak{R}^{-1}$. La dependencia de la semántica de $L_{M}$ con respecto a la semántica que corresponda a la lógica de cada uno de los mundos viene dada por (1), dado que en cada mundo se determinará qué fórmulas de $L$ son válidas (y, por tanto, las fórmulas verdaderas de $L_{M}$ serán también distintas en cada mundo). El resto de operadores de $L_{M}$ se define independientemente de estas semánticas particulares.

Intuitivamente, $\mathfrak{R} w w^{\prime}$ nos indica que desde la lógica $\pi(w)$ podemos evolucionar hacia otra lógica $\pi\left(w^{\prime}\right)$. Típicamente, si queremos modelar el razonamiento abductivo, entenderemos que $\pi\left(w^{\prime}\right)$ es una extensión de la lógica $\pi(w)$. En cuanto a los operadores modales, si dado un modelo $M$ y un mundo $w$ se cumple que en toda lógica a la que podemos evolucionar desde $\pi(w)$ se verifica $\alpha$ escribimos $(M, w) \vDash \square^{+} \alpha$. Si hay alguna lógica a la que podemos evolucionar desde $\pi(w)$ donde se verifica $\alpha$ escribimos $(M, w) \vDash \diamond^{+} \alpha$. Los operadores inversos nos hablan de las lógicas desde las cuáles podemos haber evolucionado. Por ejemplo, $(M, w) \vDash \square^{-} \alpha$ expresa que en todas las lógicas desde las cuales se puede evolucionar hacia $\pi(w)$ se verifica $\alpha$.

Visualicemos estos conceptos con un ejemplo. El siguiente grafo representa mundos y, dentro de cada uno de ellos, ciertas fórmulas verdaderas:

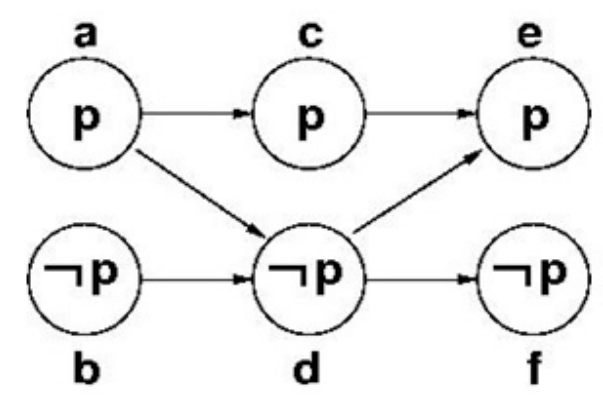

Las flechas representan la accesibilidad entre mundos. Tenemos que:

$$
\mathfrak{R}=\{(a, c),(a, d),(b, d),(c, e),(d, e),(d, f)\}
$$

Veamos, a título de ejemplo, cómo podemos evaluar algunas fórmulas: 
1. $(M, f) \vDash \square^{-}(\neg p)$; ya que todo mundo desde el que se accede a $f$ (en este caso solo $d$ ) satisface $\neg p$.

2. $(M, d) \vDash \diamond^{-}(\neg p) \& \diamond^{+} \square^{-}(\neg p) \& \sim \square^{-}(\neg p)$; ya que $d$ es accesible desde al menos un mundo (es decir, $b$ ) donde se satisface $\neg p$ y también desde $d$ es accesible un mundo (en este caso $f$ ) al cual solo se accede desde mundos (tan solo $d$ ) donde se satisface $\neg p$, y además también se da que no todo mundo desde el que $d$ es accesible satisface $\neg p$ ya que, de los dos que acceden a él ( $a$ y $b$ ), uno de ellos (precisamente $a$ ), no satisface dicha fórmula.

A continuación indicamos cómo podemos utilizar las herramientas presentadas para modelar el razonamiento abductivo.

\section{RAZONAMIENTO ABDUCTIVO EN MODELOS EXPLICATIVOS}

Para modelar el razonamiento abductivo consideramos modelos explicativos en que la relación de accesibilidad $\mathfrak{R}$ es monótona sobre el conjunto de lógicas $\Lambda$, es decir, que si tenemos $\Re w w^{\prime}$ entonces para cada $\Gamma \subseteq L$ y $\alpha \in L$ se verifica

$$
\text { Si } \Gamma \vDash_{w} \alpha \text { entonces } \Gamma \vDash_{w^{\prime}} \alpha
$$

Por tanto, cada teoría $w^{\prime}$ accesible desde una teoría $w$ lleva asociada una lógica $\pi\left(w^{\prime}\right)$ que es una extensión de $\pi(w)$, es decir, $\vDash_{w} \subseteq \vDash_{w^{\prime}}$. Así, la relación $\mathfrak{R}$ establece las posibles ampliaciones de las teorías (y $\mathfrak{R}^{-1}$ sus posibles reducciones).

Ahora podemos entender que un problema abductivo surge cuando en cierto mundo $w$ tenemos una fórmula $\phi$ que no es verdadera (no es válida en la lógica de $w$ o no se sigue de los hechos observados). Formalmente, un problema abductivo es ahora un par $\langle w, \phi\rangle$ tal que:

$$
\begin{aligned}
& \text { 1. }\langle M, w\rangle \vDash \diamond^{+} \sim \phi \\
& \text { 2. }\langle M, w\rangle \vDash \diamond^{+} \phi
\end{aligned}
$$

La primera condición indica que $\phi$ no es verdadera en todas las posibles evoluciones de la teoría $w$. Por tanto, por la propiedad de $\mathfrak{R}$ que hemos indicado más arriba, $\phi$ tampoco es verdadera en $w$. La segunda condición expresa que, sin embargo, existen teorías accesibles 
donde $\phi$ es verdadera. De este modo, estamos ante un problema abductivo, porque en la situación actual de la teoría $\pi(w)$ podemos realizar ampliaciones donde $\phi$ es verdadera (asegurado por 2) y ampliaciones donde no lo es (asegurado por 1).

La solución abductiva consiste simplemente en hacer una de las ampliaciones que verifican $\phi$ - Vendría dada por el paso a un mundo $w^{\prime}$ tal que $\mathfrak{R} w w^{\prime}$ y $\phi$ sea verdadera en $w^{\prime}$. Representamos este paso mediante una fórmula $\alpha \in L$ tal que en todas las extensiones posibles de $\pi(w)$ que hagan válida $\alpha$ se verifique $\phi$.

Por tanto, la fórmula $\alpha \in L$ será una solución abductiva a cierto problema abductivo $\langle w, \phi\rangle$ si y solo si:

1. $\langle M, w\rangle \vDash \square^{+}(\alpha \Rightarrow \phi)$

2. $\langle M, w\rangle \vDash \diamond^{+} \alpha$

3. $\langle M, w\rangle \vDash \diamond^{-} \diamond^{+}(\alpha \& \sim \phi)$

La primera condición indica que $\alpha$, junto con la teoría actual (esto es, la lógica de $w$ ) explica $\phi$, dado que afirma que en cualquier extensión de $w$ donde $\alpha$ sea válida, también lo será $\phi$. La segunda condición dice que $\alpha$ es admisible en la teoría actual, es decir, que en efecto existe una ampliación de la teoría que verifica $\alpha$. La última condición indica que $\alpha$, por sí sola, es insuficiente para explicar $\phi$, por tanto necesita la teoría actual. Obsérvese que se exige que se pueda reducir la teoría (volver a un estado anterior) de tal forma que podría evolucionarse hacia un mundo donde $\alpha$ no implicaría $\phi$.

Estos tres criterios se corresponden con los que hemos proporcionado más arriba al caracterizar la abducción consistente explicativa que para (A. Aliseda 2006) es la que posee mayor interés epistémico. Cada uno de los mundos $w^{\prime}$ a los que la segunda condición nos garantiza que podemos acceder es una teoría posible hacia la que podemos evolucionar desde $w$ para explicar $\phi$.

Las definiciones de problema abductivo y solución abductiva, en el marco de los modelos explicativos, nos proporcionan una fórmula, a saber,

$$
\left(\diamond^{+} \sim \phi\right) \&\left(\diamond^{+} \phi\right) \&\left(\square^{+}(\alpha \Rightarrow \phi)\right) \&\left(\diamond^{+} \alpha\right) \&\left(\diamond^{-} \diamond^{+}(\alpha \& \sim \phi)\right)
$$

que, cuando se verifica en $\langle M, w\rangle$, quiere decir que $\langle w, \phi\rangle$ es un problema abductivo y una de 
sus soluciones es $\alpha$. Con ello conseguimos una formalización general de la abducción. En efecto, $L_{M}$ es un lenguaje formal cuya semántica, como hemos visto, se define independientemente de cada semántica posible para $L$. Las definiciones y estipulaciones que comúnmente se establecen en términos de la correspondiente relación de consecuencia, el problema abductivo o la solución abductiva, se condensan ahora, por así decir, en la mencionada fórmula de $L_{M}$. Esto es una novedad con respecto al tratamiento de la abducción desde la lógica clásica, donde no hay ninguna fórmula semejante a la que hemos proporcionado para indicar qué son problemas abductivos y soluciones abductivas. Como vimos más arriba, en lógica clásica hay que acudir a la relación de consecuencia lógica y verificar si se da o no en ciertos casos.

Además, como nos hemos estado moviendo en un plano metateórico, en tanto que hemos trabajado con la semántica de los modelos explicativos, que es una metasemántica con respecto a la semántica de cada mundo-teoría, podemos aplicar estas definiciones a modelos explicativos concretos, estudiando así el razonamiento abductivo en relación con diversos sistemas formales.

\section{ABDUCCIÓN EN LÓGICA CLÁSICA MEDIANTE MODELOS EXPLICATIVOS}

Veamos un ejemplo concreto de nuestro modelo que permite captar el razonamiento abductivo haciendo uso de lógica clásica, tal como se presenta en (A. Aliseda 2006), si bien introducimos una ligera modificación en la noción de consecuencia lógica para hacerla relativa a un contexto. Sea $L^{\prime}$ el lenguaje de la lógica clásica de primer orden y $\vDash^{c}$ la relación de consecuencia lógica clásica. Podemos definir la relación de consecuencia módulo $\Theta$ (D. Makinson 2003), $\vDash_{\theta}^{c}$, donde $\Theta \subseteq L^{\prime}$, como

$$
\Gamma \models_{\Theta}^{c} \varphi \text { si y solo si } \Gamma \cup \Theta \vDash^{c} \varphi
$$

La relación de consecuencia módulo $\Theta$ verifica las reglas estructurales de

- Reflexividad. Para cada conjunto de sentencias $\Gamma$ y cada sentencia $\gamma \in \Gamma, \Gamma \models_{\Theta}^{c} \gamma$.

- Monotonía. Para los conjuntos de sentencias $\Gamma, \Delta$ y la sentencia $\varphi$, si $\Gamma \models_{\Theta}^{c} \varphi$, entonces $\Gamma \cup \Delta \vDash_{\Theta}^{c} \varphi$,

- Corte. Para los conjunto de sentencias $\Gamma, \Delta y$ las sentencias $\varphi, \psi$, si $\Gamma \vDash_{\Theta}^{c} \varphi$ y 


$$
\Delta \cup\{\varphi\} \vDash_{\Theta}^{c} \psi \text {, entonces } \Delta \cup \Gamma \vDash_{\Theta}^{c} \psi \text {. }
$$

Los tres casos se demuestran fácilmente, tomando como base la definición de $\vDash_{\theta}^{c}$ proporcionada. Por otra parte, estas relaciones cumplen compacidad. Sin embargo no se pueden reducir meramente a la relación de consecuencia lógica clásica pues, a diferencia de ésta última, aquellas no verifican sustitución uniforme. En efecto, si $\sigma$ representa la operación estándar de sustitución y, para cada conjunto no vacío de sentencias $\Gamma$, definimos $\sigma(\Gamma)=\{\sigma(\alpha) \in L \mid \alpha \in \Gamma\}$, entonces, dada $\vDash_{\Theta}^{c}$, es posible que $\Gamma \vDash_{\Theta}^{c} \varphi$ pero $\sigma(\Gamma) \not \nvdash_{\Theta}^{c} \sigma(\varphi)$, pues el módulo $\Theta$ no se ve afectado por la sustitución $\sigma$.

Tal como se define la noción de solución abductiva en lógica clásica no se contempla la posibilidad de extensión de la lógica subyacente, sino la adición de una fórmula $\alpha$ a una teoría $\Theta$ para que se verifique $\Theta \cup\{\alpha\} \vDash \phi$. Se trata de una caracterización distinta de la que hemos mostrado en la sección anterior, dado que no hay cambio de lógica sino extensión de la teoría. Sin embargo, desde la generalidad de los modelos explicativos podemos dar cuenta también del modelo clásico de razonamiento abductivo. Para hacerlo, entenderemos cada mundo $w$ como un conjunto de fórmulas. Entonces, asignamos a cada mundo $w$ la lógica dada por la relación de consecuencia módulo $w$, es decir, $\vDash_{w}^{c}$. Así, hacemos que la teoría base (ahora, el conjunto de fórmulas $w$ ) de las definiciones clásicas se convierta en la lógica subyacente (ahora, $\models_{w}^{c}$ ). Así, solo tendremos que garantizar que si $\mathfrak{R} w w^{\prime}$ con $\pi(w)=\left\langle L^{\prime}, \models_{w}^{c}\right\rangle$ y $\pi\left(w^{\prime}\right)=\left\langle L^{\prime}, \models_{w^{\prime}}^{c}\right\rangle$, entonces $w \subseteq w^{\prime}$. Formalmente, el modelo explicativo $M^{c}$ se define como

$$
M^{c}=\left\langle L^{\prime}, \Lambda, W, \mathfrak{R}, \pi\right\rangle
$$

donde:

- $\quad L^{\prime}$, como se ha explicado, es el lenguaje de la lógica clásica de primer orden.

- $\quad W$ es la familia de los subconjuntos consistentes de $L^{\prime}$.

- $\Lambda=\left\{\left\langle L^{\prime}, \models_{w}^{c}\right\rangle \mid w \in W\right\}$

- $\pi(w)=\left\langle L^{\prime}, \models_{w}^{c}\right\rangle$

- $\mathfrak{R}=\left\{\left\langle w, w^{\prime}\right\rangle \in W^{2} \mid w \subseteq w^{\prime}\right\}$

La semántica se define tal como vimos anteriormente, teniendo en cuenta que ahora, dada 
$\varphi \in L^{\prime}:$

$$
\|\varphi\|=\left\{w \in W: \vDash_{w}^{c} \varphi\right\}
$$

El modelo $M^{c}$ representa un conjunto de teorías clásicas, y los problemas abductivos en este modelo se corresponden con la noción de abducción en lógica clásica, pero expresada dentro del formalismo propuesto.

Tal como se ha definido la relación de accesibilidad, resulta ser reflexiva y transitiva, aunque no simétrica. De aquí que son válidos los axiomas y reglas del sistema modal S4. De este modo, el análisis estructural del razonamiento abductivo, generalmente dado en términos metalógicos, se puede construir en el lenguaje $L_{M}$.

\section{CONCLUSIONES}

Como se observa en el ejemplo de $M^{c}$, nuestro acercamiento permite introducir en el ámbito formal caracterizaciones del razonamiento abductivo que de otro modo solo eran posibles dentro de la metalógica no formalizada. Igualmente, al estudiarse el cambio epistémico al nivel de $L_{M}$, y no de $L$, queda abierta la puerta a estudios generales sobre la evolución de las teorías más allá de los habituales tratamientos del razonamiento abductivo centrados en una lógica particular. Ahora, la abducción ocurre en el terreno de $L_{M}$ y la deducción en el de $L$, por así decir. Ambos ámbitos tienen semánticas definidas separadamente. Uno de los objetivos de (J. Hintikka 1998) era independizar el razonamiento abductivo del deductivo. Queda abierta la pregunta de hasta qué punto puede ser esto logrado siguiendo nuestra propuesta.

Las redes teóricas estudiadas por el estructuralismo contienen un conjunto de elementos teóricos. Entre tales elementos se establece una relación de especialización entre modelos. Las características concretas de esta relación de especialización, en ciertos casos, se pueden hacer corresponder con una relación de accesibilidad. De esta manera, los modelos explicativos propuestos pueden ofrecer nuevos elementos para los estudios acerca de la concepción estructural de las teorías científicas y sus aplicaciones. 


\section{REFERENCIAS}

ALCHOURRÓN, C.E.; GÄRDENFORS, P.; MAKINSON, D. 1985: «On the logic of theory change: partial meet contraction and revision functions», Journal of Symbolic Logic, 50(2), pp. 510-530.

ALISEDA, A. 2006: Abductive Reasoning: Logical Investigations into Discovery and Explanation, Dordrecht: Springer.

DÍEZ, J.A.; MOULINES, C.U. 1997: Fundamentos de Filosofía de la Ciencia, Barcelona, Ariel.

HINTIKKA, J. 1998: «What is abduction? The fundamental problem of contemporary epistemology», Transactions of the Charles S. Peirce Society, 34(3), pp. 503-533.

LIPTON, P. 1991: Inference to the Best Explanation, Londres: Routledge.

MAKINSON, D. 2003: «Bridges between classical and nonmonotonic logic», Logic Journal of the IGPL, 11(1), pp. 69-96.

MARTÍNEZ, S.F. 1995: «Lógica cuántica», en C.E. Alchourrón (ed.) Lógica. Enciclopedia Iberoamericana de Filosofía, Madrid: Trotta, pp. 227-236.

MAYER, M.C.; PIRRI, F. 1993: «First order abduction via tableau and sequent calculi», Bulletin of the IGPL, 1, pp. 99-117.

ROBINSON, A. 1966: Non-Standard Analysis, Amsterdam: North-Holland Publishing Company.

ZABALARDO, J.L. 2002: Introducción a la teoría de la lógica, Madrid: Alianza. 